\title{
Co-regular Edge Domination in Graphs
}

\author{
M. H. Muddebihal \\ Professor \\ Department of Mathematics \\ Gulbarga University \\ Kalaburagi-585106 \\ Karnataka, India
}

\author{
Priyanka H. Mandarvadkar \\ Research Scholar \\ Department of Mathematics \\ Gulbarga University \\ Kalaburagi-585106 \\ Karnataka, India
}

\begin{abstract}
An edge dominating set $D$ is a coregular edge dominating set of $G$. If the induced subgraph $\langle E(G)-D\rangle$ is regular. The coregular edge domination number $\gamma_{c r}^{\prime}(G)$ is the minimum cardinality of a coregular edge dominating set. We establish upper and lower bounds on $\gamma_{c r}^{\prime}(G)$ and compare with other dominating parameters $G$ and elements of $G$ were obtained.
\end{abstract}

\section{Keywords}

Graph, Edge domination number, Coregular edge domination number.

\section{Subject classified number: AMS-05C69, $05 \mathrm{C70}$.}

\section{INTRODUCTION}

By a graph $G=(V, E)$ be mean of finite undirected graphs without loops or multiple edges. Terms not here are used in the sence of Harary [7].

As usual the maximum degree of a vertex in $V(G)$ is denoted by $\Delta(G)$ and maximum edge degree of edge in $E(G)$ is denoted by $\Delta^{\prime}(G)$. The notation $\alpha_{0}(G)\left(\alpha_{1}(G)\right)$ is the minimum number of vertices(edges) in vertex(edge) cover of $G$. The notation $\beta_{0}(G)\left(\beta_{1}(G)\right)$ is the maximum cardinality of a vertex(edge) independent set in G. A subset $D$ of $V$ is a dominating set of $G$, if every vertex not in $D$ is adjacent to some vertex in D. The domination number $\gamma(G)$ of $G$ is the minimum cardinality taken over all dominating sets of $G$. The study of domination in graphs was begun by Ore[15] and Berge[4].

We begin by recalling some standard definitions from domination theory.

A dominating set $\mathrm{S}$ of $\mathrm{G}$ is said to be a connected dominating set if the subgraph $\langle\mathrm{S}\rangle$ is connected in $\mathrm{G}$. The minimum cardinality of vertices in such a set is called the connected domination number, of $G$ and is denoted by $\gamma_{c}(G)$.

A dominating set $\mathrm{S}$ of $\mathrm{G}$ is said to be a total dominating set if the subgraph $\langle\mathrm{S}\rangle$ has no isolated vertices in $\mathrm{G}$. The minimum cardinality of vertices in such a set is called the total domination number, denoted by $\gamma_{\mathrm{t}}(\mathrm{G})$ see [5].

The concept of restrained domination in graphs was introduced by Domke et.al (1999) see [6]. A dominating set $S \subseteq V(G)$ is restrained dominating set of $G$, if every vertex not in $S$ is adjacent to a vertex in $S$ and to a vertex in $V(G)-$ $S$. The restrained domination number of a graph $G$ is denoted by $\gamma_{r}(G)$ is the minimum cardinality of a restrained dominating set in $G$.

A dominating set $\mathrm{D}$ of a graph $\mathrm{G}=(\mathrm{V}, \mathrm{E})$ is a split dominating set if the induced subgraph $\langle\mathrm{V}-\mathrm{D}\rangle$ has more than one component. The split domination number $\gamma_{s}(G)$ of $G$ is the least cardinality of a split dominating set. The concept of domination was introduced in [10].

A restrained dominating set $\mathrm{D} \subseteq \mathrm{V}(\mathrm{G})$ is a coregular restrained dominating set if the induced subgraph $\langle\mathrm{V}-\mathrm{D}\rangle$ is regular. The coregular restrained domination number of $G$ is denoted by $\gamma_{\mathrm{crr}}(\mathrm{G})$ is the minimum cardinality of a coregular restrained dominating set. For detail see [13].

A dominating set $\mathrm{D}$ of a graph $\mathrm{G}$ is a global dominating set if $D$ is also a dominating set of $\bar{G}$. The global domination number $\gamma_{\mathrm{g}}(\mathrm{G})$ is the minimum cardinality of a global dominating set of $\mathrm{G}$.

The concept of Roman domination function (RDF) in a graph $\mathrm{G}=(\mathrm{V}, \mathrm{E})$ is a function $\mathrm{f}: \mathrm{V} \rightarrow\{0,1,2\}$ satisfying the condition that every vertex $u$ for which $f(u)=0$ is adjacent to at least one vertex of $v$ for which $f(v)=2$ in $G$. The weight of a Roman dominating function is the value $f(V)=\sum_{u \in V} f(u)$. The minimum weight of a Roman domination function of a graph $\mathrm{G}$ is called Roman domination number and is denoted by $\gamma_{R}(G)$.

A dominating set $\mathrm{D} \subseteq \mathrm{V}(\mathrm{G})$ is a double dominating set of $\mathrm{G}$, if each vertex in $\mathrm{V}$ is dominated by at least two vertices in $\mathrm{D}$. The double domination number $\gamma_{\mathrm{dd}}(\mathrm{G})$ of $\mathrm{G}$ is the minimum cardinality of a double dominating set of $\mathrm{G}$ see [9].

Analogously, a split dominating set D of a graph G is a coregular split dominating set if the induced subgraph $<\mathrm{V}(\mathrm{G})-\mathrm{D}\rangle$ is disconnected and regular. The coregular split dominating number $\gamma_{\mathrm{crs}}(\mathrm{G})$ is the minimum cardinality of a coregular split dominating set of G. For details see [12].

A total dominating set $\mathrm{D}$ of a graph $\mathrm{G}$ is a coregular total dominating set if the induced subgraph $\langle\mathrm{V}-\mathrm{D}\rangle$ is regular. The coregular total domination number $\gamma_{\mathrm{crt}}(\mathrm{G})$ of $\mathrm{G}$ is the minimum cardinality of a coregular total dominating set see [14].

The concept of edge domination was introduced and studied in $[2,11]$.

In this paper, we obtain many bounds on $\gamma_{\mathrm{cr}}^{\prime}(G)$ in terms of elements of $G$. Also its relation with other domination parameters were established.

We need the following theorem for our further results.

Theorem A [1]: Let $\mathrm{G}$ be a connected graph of order $\mathrm{n}$, then $\gamma^{\prime}(G) \leq\left[\frac{\mathrm{n}}{2}\right]$.

\section{MAIN RESULTS}

Theorem 2.1 a]. For any path $P_{p}$ with $p \geq 3$ vertices, 


$$
\gamma_{\mathrm{cr}}^{\prime}\left(\mathrm{P}_{\mathrm{p}}\right)=\left\lceil\frac{\mathrm{p}}{2}\right\rceil-1 \text {. }
$$

b]. For any cycle $C_{p}$ with $p \geq 3$ vertices,

$$
\gamma_{\mathrm{cr}}^{\prime}\left(\mathrm{C}_{\mathrm{p}}\right)=\left\lceil\frac{\mathrm{p}}{2}\right\rceil .
$$

c]. For any $\operatorname{star} K_{(1, p)}$ with $p \geq 3$ vertices,

$$
\gamma_{\mathrm{cr}}^{\prime}\left(\mathrm{K}_{1, \mathrm{p}}\right)=\mathrm{q}-1
$$

d]. For any wheel $W_{p}$ with $p \geq 4$ vertices,

$$
\gamma_{\mathrm{cr}}^{\prime}\left(\mathrm{W}_{\mathrm{p}}\right)=\mathrm{p}-1 \text {. }
$$

Theorem 2.2: For any connected $(p, q)$ graph $G$ with $p \geq 3$ vertices,

$\gamma_{\mathrm{cr}}^{\prime}(G)+m \geq \beta_{0}(G) \quad$ where $m$ be the number of end vertices in $\mathrm{G}$

Proof: Let $\mathrm{E}=\left\{\mathrm{e}_{1}, \mathrm{e}_{2}, \ldots \ldots \ldots, \mathrm{e}_{\mathrm{k}}\right\}$ be the edge set in G. Now consider $\mathrm{E}_{1}=\left\{\mathrm{e}_{1}, \mathrm{e}_{2}, \ldots . ., \mathrm{e}_{\mathrm{m}}\right\} \subseteq \mathrm{E}(\mathrm{G})$ be the set of edges with maximum edge degree and $\mathrm{E}_{2}=\left\{\mathrm{e}_{1}, \mathrm{e}_{2}, \ldots \ldots, \mathrm{e}_{\mathrm{n}}\right\} \subseteq$ $\mathrm{E}(\mathrm{G})$ be the set of edges with minimum edge degree. Suppose $\mathrm{E}_{1}{ }^{\prime} \subseteq \mathrm{E}_{1}$ and $\mathrm{E}_{2}{ }^{\prime} \subseteq \mathrm{E}_{2}$ then $\left\{\mathrm{E}_{1}{ }^{\prime} \cup \mathrm{E}_{2}{ }^{\prime}\right\}$ forms a minimal edge dominating set of $\mathrm{G}$. Further if induced subgraph $<\mathrm{E}(\mathrm{G})-\left\{\mathrm{E}_{1}{ }^{\prime} \cup \mathrm{E}_{2}^{\prime}\right\}>$ is regular then $\left\{\mathrm{E}_{1}{ }^{\prime} \cup \mathrm{E}_{2}{ }^{\prime}\right\}$ itself is a coregular edge dominating set of $\mathrm{G}$. On the other hand let $A=\left\{v_{1}, v_{2}, \ldots \ldots, v_{n}\right\}$ be the set of all endvertices in G. Let $\mathrm{K}=\left\{\mathrm{v}_{1}, \mathrm{v}_{2}, \ldots \ldots \ldots, \mathrm{v}_{\mathrm{p}}\right\} \subseteq \mathrm{V}(\mathrm{G})$ be the maximum set of vertices such that $\operatorname{deg}\left(\mathrm{v}_{\mathrm{i}}, \mathrm{v}_{\mathrm{j}}\right) \geq 2$, and $\mathrm{N}\left(\mathrm{v}_{\mathrm{i}}\right) \cap \mathrm{N}\left(\mathrm{v}_{\mathrm{j}}\right)=$ $\mathrm{x}, \forall \mathrm{v}_{\mathrm{i}}, \mathrm{v}_{\mathrm{j}} \in \mathrm{K}$ so that $\mathrm{x} \in \mathrm{V}(\mathrm{G})-\mathrm{K}$. Clearly $|\mathrm{K}|=\beta_{\circ}(\mathrm{G})$. It follows that $\left|\left\{E_{1}{ }^{\prime} \cup E_{2}{ }^{\prime}\right\}\right|+|A| \geq|K|$ which gives, $\gamma_{\mathrm{cr}}^{\prime}(\mathrm{G})+\mathrm{m} \geq \beta_{0}(\mathrm{G})$.

Theorem 2.3: For any connected $(p, q)$ graph $G$ with $p \geq 4$ vertices, $P_{4}$.

$$
\gamma_{c r}^{\prime}(G) \geq \gamma_{c r t}(G)+\gamma_{s}(G)-\gamma_{c}(G) \text { with, } G \neq K_{p}, G \neq
$$

Proof: Let $E_{1}=\left\{e_{1}, e_{2}, \ldots \ldots, e_{k}\right\} \subseteq E(G)$ be the minimal set of edges which covers all the edges in $G$ such that $N\left[E_{1}\right]=$ $E(G)$. Then $E_{1}$ is the edge dominating set of $G$. If the induced subgraph $\left\langle E(G)-E_{1}\right\rangle$ is regular then $E_{1}$ is a coregular edge dominating set of $G$. Suppose $A=\left\{v_{1}, v_{2}, \ldots ., v_{m}\right\} \subseteq$ $V(G)$ such that $\operatorname{deg}\left(v_{j}\right) \geq 2,1 \leq j \leq m$. Then there exists at least one vertex $v$ of maximum degree of $G$ in $A$. Let $D$ be a minimal dominating set of $G$ such that $D \subseteq A$ if the subgraph $<D>$ has exactly one component then $D$ itself is a connected dominating set of $G$. On the other hand if the induced subgraph $\langle V(G)-D\rangle=F$ is disconnected then $\{F\}$ is a split dominating set of $G$. Further $V(G)-D=B, \forall v_{i} \in B$ if $<D \cup\left\{v_{i}\right\}>$ has no isolates. Then $\left\langle D \cup\left\{v_{i}\right\}>\right.$ forms a minimal total dominating set of $G$. Also if $B_{1}=[V(G)-D \cup$ $\left.\left\{v_{i}\right\}\right]$ and $\forall v_{i} \in<B_{1}>$ has same degree then $\left\{B_{1}\right\}$ is a $\gamma_{c r t}-$ set of $G$. It follows that $\left|E_{1}\right| \geq|B|+|F|-D$, which gives $\gamma_{c r}^{\prime}(G) \geq \gamma_{c r t}(G)+\gamma_{s}(G)-\gamma_{c}(G)$.

Theorem 2.4: For any connected $(p, q)$ graph $G$ with $p \geq 3$ vertices,

$G \neq P_{p},(p \leq 6)$

$$
\gamma_{c r}^{\prime}(G) \geq \gamma_{c r r}(G)-\gamma(G)+2 \text { and } G \neq K_{1, p},
$$

Proof: For the graph $G=P_{p}$ with $p \leq 6$ For $p=4$, $\gamma_{c r}^{\prime}(G)=1<\gamma_{c r r}(G)-\gamma(G)+2=2 . \quad$ For $\quad p=5,6$, $\gamma_{c r}^{\prime}(G)=2<(G)-\gamma(G)+2=3,4$ and hence the result not holds for path $p \leq 6$. Let $A=\left\{v_{1}, v_{2}, \ldots \ldots, v_{p}\right\} \subseteq V(G)$ be set of vertices with $\operatorname{deg}\left(v_{i}\right) \geq 1$, such that $N[A]=V(G)$. Clearly $A$ forms a dominating set of $G$. Suppose $B=$ $\left\{v_{1}, v_{2}, \ldots \ldots, v_{k}\right\} \subseteq V(G)$ be the set of endvertices in $G$ and $A^{\prime}=V(G)-B$. Then there exists a vertex set $H \subseteq A^{\prime}$ such that $\forall v_{i} \in\{V(G)-H \cup B\}$ is adjacent to at least one vertex of $\{H \cup B\}$ and in $V(G)-H \cup B$. Then $\{H \cup B\}$ is a $\gamma_{r}$ set of $G$. If $\langle V(G)-\{H \cup B\}>$ is regular then $\{H \cup B\}$ itself is a $\gamma_{c r r}$ set of $G$. Let $\left\{e_{1}, e_{2}, \ldots \ldots \ldots, e_{p}\right\}=E(G)$ be the edge set in $G$. Suppose $S$ be the minimal edge dominating set of $G$. If $<E(G)-S>$ has same degree then $S$ itself is a $\gamma_{c r}^{\prime}$ set of $G$. Hence $|S| \geq|\{H \cup B\}|+|A|+2$ which gives, $\gamma_{c r}^{\prime}(G) \geq$ $\gamma_{c r r}(G)-\gamma(G)+2$

Theorem 2.5: For any connected $(p, q)$ graph $G$ with $p \geq 4$ vertices,

$$
\gamma_{c r}^{\prime}(G) \geq \alpha_{0}(G)-\gamma_{c r s}(G)+2 \text { with } G \neq K_{p}, G \neq P_{4}
$$

Proof: Suppose $G=K_{p}$. Then by the definition $\gamma_{s}$ set does not exists, hence $\gamma_{c r s}$ also does not exists. Let $D=$ $\left\{v_{1}, v_{2}, \ldots \ldots \ldots, v_{p}\right\}$ be the minimal set of vertices in $G$, such that $\langle V(G)-D\rangle$ is regular and which gives more than one component. Then $D$ forms a minimal coregular split dominating set of $G$. Suppose $B=\left\{v_{1}, v_{2}, \ldots \ldots, v_{n}\right\} \subseteq V(G)$ $\forall e_{i} \in E(G)$ is incident to at least one vertex $B$. Then $|B|=\alpha_{0}(G)$. Further $E(G)=\left\{e_{1}, e_{2}, \ldots \ldots, e_{n}\right\}$ be the edge set of $G$. Let $A=\left\{e_{1}, e_{2}, \ldots \ldots, e_{k}\right\} \subseteq E(G)$ which covers all the edges in $G$. Such that $N[A]=E(G)$, then $A$ is a minimal edge dominating set of $G$. If the induced subgraph $<E(G)-$ $A>$ has same degree then $A$ is a $\gamma_{c r}^{\prime}$ set of $G$. It follows that $|A| \geq|B|-|D|+2$. Which gives, $\quad \gamma_{c r}^{\prime}(G) \geq \alpha_{0}(G)-$ $\gamma_{\text {crs }}(G)+2$.

Corollary 2.1: For any connected $(p, q)$ graph $G \gamma_{c r}^{\prime}(G) \geq$ $\gamma^{\prime}(G)$.

Theorem 2.6: For any connected $(p, q)$ graph $G$ with $p \geq 3$ vertices,

$$
2 \gamma_{c r}^{\prime}(G) \geq \gamma^{\prime}(G)+\gamma_{s}(G) \text { and } G \neq K_{p}, G \neq P_{4} .
$$

Proof: Suppose $G=K_{p}$ by the definition, $\gamma_{s}$-set does not exists. Also if $G=p_{4}$, then $2 \gamma_{c r}^{\prime}(G)<\gamma^{\prime}(G)+\gamma_{s}(G)$, a contradiction to $P_{4}$. Let $A=\left\{v_{1}, v_{2}, \ldots \ldots ., v_{p}\right\} \subseteq V(G)$ be the set of all endvertices in $G$ and $A^{\prime}=V(G)-A$. Suppose there exists a vertex set $B \subset A^{\prime}$, such that $D=[V(G)-B]$ is a dominating set of $G$. Hence $\langle D\rangle$ has more than one component then $D$ forms a $\gamma_{s}$ - set of $G$. Further let $E=$ $\left\{e_{1}, e_{2}, \ldots \ldots \ldots, e_{p}\right\}$ be the edge set in $G$. Now consider $E_{1}=\left\{e_{1}, e_{2}, \ldots \ldots \ldots, e_{m}\right\} \subseteq E(G)$ be the set of edges with maximum edge degree and $E_{2}=\left\{e_{1}, e_{2}, \ldots \ldots \ldots, e_{n}\right\} \subseteq E(G)$ be the set of edges with with minimum edge degree. Suppose $E_{1}^{\prime} \subseteq E_{1}$ and $E_{2}^{\prime} \subseteq E_{2}$ if every edge in $\left\{E_{1}^{\prime} \cup E_{2}^{\prime}\right\}$ is adjacent to an edge in $\left\{V(G)-E_{1}^{\prime} \cup E_{2}^{\prime}\right\}$ then $\left\{E_{1}^{\prime} \cup E_{2}^{\prime}\right\}$ for a $\gamma^{\prime}-$ set of $G$. Suppose $\left\{V(G)-E_{1}^{\prime} \cup E_{2}^{\prime}\right\}=S$ is regular. Clearly $\{S\}$ is a $\gamma_{c r}^{\prime}$ - set of $G$. Thus $2|S| \geq\left|E_{1}^{\prime} \cup E_{2}^{\prime}\right|+|D|$ which gives, $2 \gamma_{c r}^{\prime}(G) \geq \gamma^{\prime}(G)+\gamma_{s}(G)$.

Theorem 2.7: For any connected $(p, q)$ graph $G$ with $p \geq 3$ vertices,

$$
\begin{aligned}
& 2 \gamma_{c r}^{\prime}(G) \geq \alpha_{1}(G)-\Delta^{\prime}(G)+\gamma_{g}+1 \quad \text { with } \quad G \neq \\
& P_{p}(P \leq 4)
\end{aligned}
$$


Proof: Let $E=\left\{e_{1}, e_{2}, \ldots \ldots \ldots, e_{k}\right\}$ be the edge set of $G$. Suppose $E^{\prime} \subseteq E$ then $N\left[E^{\prime}\right]=E(G)$ then $E^{\prime}$ is an edge dominating set of $G$. If $\left\langle E(G)-\left\{E^{\prime}\right\}>\right.$ is a regular, then $\left\{E^{\prime}\right\}$ itself is a $\gamma_{c r}^{\prime}$ set of $G$. Let $e$ be an edge with degree $\Delta^{\prime}$ and let $D=\left\{v_{1}, v_{2}, \ldots \ldots, v_{n}\right\} \subseteq V(G)$ and $D \subseteq V(\bar{G})$. If $N[D]=V(G)$ and $N[D]=V(\bar{G})$. Then $D$ is a dominating set of $G$ and $\bar{G}$. Let $A=\left\{e_{1}, e_{2}, \ldots \ldots . e_{m}\right\}$ be the set of all endedges in $G$. Then $A \cup F$ where $F \subseteq E(G)-A$ be the minimal set of edges which covers all the vertices of $G$ such that $|A \cup F|=\alpha_{1}(G)$. Thus $2\left|\left\{E^{\prime}\right\}\right| \geq|A \cup F|-|e|+|D|+$ 1 which gives, $2 \gamma_{c r}^{\prime}(G) \leq \alpha_{1}(G)-\Delta^{\prime}(G)+\gamma_{g}+1$.

Theorem 2.8: For any connected $(p, q)$ graph $G$ with $p \geq 3$ vertices,

$$
\gamma_{c r}^{\prime}(G)+\operatorname{diam}(G)+\gamma(G) \geq \gamma_{R}(G)+\gamma_{t}(G) .
$$

Proof: Let $B \subseteq V(G)$ be the minimal set of vertices. Further, there exists an edge set $J \subseteq J^{\prime}$ where $J^{\prime}$ is the set of edges which are incident with the vertices of $B$ constituting the longest path in $G$ such that $|J|=\operatorname{diam}(G)$. Let $D=$ $\left\{v_{1}, v_{2}, \ldots \ldots, v_{n}\right\} \subseteq B$ be the minimal set of vertices which covers all the vertices in $G$. Clearly $D$ forms a dominating set of $G$. Suppose the subgraph $\langle D\rangle$ has no isolates. Then $D$ itself is a $\gamma_{t}(G)$ set. Otherwise if $\operatorname{deg}\left(v_{k}\right)<1$ then attach the vertices $w_{i} \in N\left(v_{k}\right)$ to make $\operatorname{deg}\left(v_{k}\right) \geq 1$ such that $<D \cup$ $\left\{w_{i}\right\}>$ does not contain any isolated vertex. Clearly $D \cup\left\{w_{i}\right\}$ forms a total dominating set of $G$. Further let function $f: V(G) \rightarrow\{0,1,2\}$ and partition the vertex set $V(G)$ into $\left(V_{0}, V_{1}, V_{2}\right)$ induced by $f$ with $\left|V_{i}\right|=n_{i}$ for $i=0,1,2$. Suppose the set $V_{2}$ dominates $V_{0}$. Then $S=V_{1} \cup V_{2}$ forms a minimal Roman dominating set of $G$. Further let $A=$ $\left\{e_{1}, e_{2}, \ldots \ldots \ldots, e_{p}\right\} \subseteq E(G)$ be the minimal set of edges which covers all the edges in $G$. Clearly $A$ forms a minimal edge dominating set of $G$. If $\langle E(G)-A\rangle$ is regular then $A$ is a coregular edge dominating set of $G$. Then $|A|+|J|+|D| \geq$ $|S|+\left|D \cup\left\{w_{i}\right\}\right|$ which gives, $\gamma_{c r}^{\prime}(G)+\operatorname{diam}(G)+\gamma(G) \geq$ $\gamma_{R}(G)+\gamma_{t}(G)$.

In the following theorem we establish the relationship between $\gamma_{d d}(G), \gamma_{r}(G)$ with coregular edge domination of a graph $G$.

Theorem 2.9: For any connected $(p, q)$ graph $G$ with $p \geq 3$ vertices,

$$
\gamma_{c r}^{\prime}(G)+\gamma_{d d}(G) \geq\left\lfloor\frac{p}{2}\right\rfloor+\gamma_{r}(G)-1 .
$$

Proof: Let $S=\left\{e_{1}, e_{2}, \ldots \ldots ., e_{m}\right\}$ be an edge dominating set of $G$. Let $D_{1}=\left\{v_{1}, v_{2}, \ldots \ldots \ldots, v_{k}\right\}$ which is dominating set of $G$. Suppose $V_{1} \subseteq V(G)-D_{1}$ be the set of vertices which are neighbours of the elements of $D_{1}$. Further $D_{2} \subseteq V_{2}$ and $D_{2} \in N\left(D_{1}\right)$. Then $D^{d}=D_{1} \cup D_{2}$ forms double dominating set of $G$ such that any vertex $v \in V(G)-D^{d}$ has at least two neighbours in $D_{1} \cup D_{2}$. Further let $A=\left\{e_{1}, e_{2}, \ldots \ldots, e_{p}\right\} \subseteq$ $E(G)$ be the minimal set of edges which covers all the edges in $G$. Such that $N\left[E_{1}\right]=E(G)$. Then $E_{1}$ is an edge dominating set of $G$. If $<E(G)-E_{1}>$ is regular then $\left\{E_{1}\right\}$ itself is a $\gamma_{c r}^{\prime}$ set of $G$. Let $B=\left\{v_{1}, v_{2}, \ldots \ldots, v_{p}\right\} \subseteq V(G)$ be the set of endvertices in $G$ and $B^{\prime}=V(G)-B$. Then there exists vertex set $H \subseteq B^{\prime}$ such that $\forall v_{i} \in\{V(G)-\{H \cup B\}$ is adjacent to at least one vertex of $\{H \cup B\}$ and in $V(G)-\{H \cup$ $B\}$. Then $\{H \cup B\}$ is a $\gamma_{r}$ set of $G$. Also by theorem $A, \gamma^{\prime}(G) \leq$ $\left|\frac{p}{2}\right|$. Thus $\left|E_{1}\right|+\left|D^{d}\right| \geq\left|\frac{V(G)}{2}\right|+|H \cup B|-1$ which gives, $\gamma_{c r}^{\prime}(G)+\gamma_{d d}(G) \geq\left\lfloor\frac{p}{2}\right\rfloor+\gamma_{r}-1$.
Theorem 2.10: For any connected $(p, q)$ graph $G$ with $p \geq 3$ vertices,

$$
\gamma_{c r}^{\prime}(G)+\gamma_{e}^{\prime}(G)+1 \leq 2(p-1) .
$$

Proof: Suppose $D$ be a minimal edge dominating set of $G$ and $E(G)-D$ be the set of all edges which are adjacent to the edges in $D$. Then $D^{\prime}=[E(G)-D]$ has same degree then $\left\{D^{\prime}\right\}$ is a coregular edge dominating set of $G$. Now let $E_{1}=$ $\left\{e_{1}, e_{2} \ldots \ldots \ldots, e_{j}\right\}$ denote the set of all endedges in $G$ and $E_{2}=E(G)-E_{1}$. Further if $F \subseteq E_{2}$ is edge dominating set of subgraph $\left\langle E_{2}\right\rangle$ then $E_{1} \cup F$ forms an endedge dominating set of $G$. Clearly it follows that $\left|D^{\prime}\right|+\left|E_{1} \cup F\right|+1 \leq 2(p-$ 1) and hence $\gamma_{c r}^{\prime}(G)+\gamma_{e}^{\prime}(G)+1 \leq 2(p-1)$.

An edge dominating set $X$ is called a connected edge dominating set if the edge induced subgraph $\langle X\rangle$ is connected. The minimum cardinality of a connected edge dominating set of $G$ is called the connected edge domination number of $G$ and is denoted by $\gamma_{c}^{\prime}(G)$. For detail see [3].

Theorem 2.11: For any connected graph $G$ with $p \geq 4$ vertices,

$$
\underset{P_{p}(p \leq 5)}{\gamma_{c r}^{\prime}(G)+\gamma_{c}^{\prime}(G) \geq \alpha_{1}(G)+\gamma_{s}(G) \quad \text { and } \quad G \neq K_{p}, G \neq}
$$

Proof: For the graph $G=P_{p}$ with $p \leq 5$ if $p=3,4,5$ then $\gamma_{c r}^{\prime}(G)+\gamma_{c}^{\prime}(G)=2,2,4 \ngtr \alpha_{1}(G)+\gamma_{s}(G)=3,4,5$. Hence $G \neq P_{p}$ with $p \leq 5$. Suppose $D=\left\{e_{1}, e_{2}, \ldots \ldots, e_{n}\right\}$ be the set of all endedges in $G$. Then $D \cup J$ where $J \subseteq E(G)-D$ be the minimal set of edges which covers all the vertices of $G$ such that $|D \cup J|=\alpha_{1}(G)$. Let $D_{1}=\left\{e_{1}, e_{2}, \ldots \ldots \ldots, e_{j}\right\}$ be the set of nonendedges which covers all the edges in $G$. If the induced subgraph $\left\langle E(G)-D_{1}>\right.$ is regular then $\left\{D_{1}\right\}$ is a coregular edge dominating set of $G$. Now consider $S=\left\{e_{1}, e_{2}, \ldots \ldots, e_{i}\right\}$ be the minimal edge dominating set then $\langle S\rangle$ does not contain more than one component. Then $S$ itself is a connected edge dominating set of $G$. Otherwise if the subgraph $\langle S\rangle$ has more than one component then attach the minimum number of edges $\left\{e_{k}\right\} \in E(G)-S$ with $\operatorname{deg}\left(e_{k}\right) \geq$ 2 such that $S_{1}=S \cup\left\{e_{k}\right\}$ forms exactly one component clearly $S_{1}$ forms a $\gamma_{c}^{\prime}$ set of $G$. On the other hand let $F=$ $\left\{v_{1}, v_{2}, \ldots \ldots \ldots, v_{n}\right\}$ be a minimal dominating set $G$ if the $\langle V(G)-F\rangle$ is disconnected then clearly $F$ forms a split dominating set of $G .\left|D_{1}\right|+\left|S_{1}\right| \geq|D \cup J|+|F|$ which gives, $\gamma_{c r}^{\prime}(G)+\gamma_{c}^{\prime}(G) \geq \alpha_{1}(G)+\gamma_{s}(G)$.

Theorem 2.12: For any connected $(p, q)$ graph $G$ with $p \geq 3$ vertices,

$$
\gamma_{c r}^{\prime}(G)+\gamma(G)+1 \leq p+\gamma_{c}^{\prime}(G) .
$$

Proof: Let $D$ be a dominating set of $G$ and let $E=$ $\left\{e_{1}, e_{2}, \ldots \ldots, e_{n}\right\} \subseteq E(G)$ be the set of all nonendedges in $G$. Suppose there exist a minimal set of edges such that $N\left[e_{i}\right]=$ $E(G), \forall e_{i} \in E_{1}, 1 \leq i \leq n$ then $E_{1}$ forms a minimal edge dominating set of $G$. Further if subgraph $\left\langle E_{1}\right\rangle$ has exactly one component then $E_{1}$ itself is a connected edge dominating set of $G$. Further $E_{2} \subseteq E_{1}$ such that the induced subgraph $\left\langle E(G)-E_{2}\right\rangle$ is regular clearly $E_{2}$ is a coregular edge dominating set of $G$. Hence $\left|E_{2}\right|+|D|+1 \leq|V(G)|+\left|E_{1}\right|$ which gives, $\quad \gamma_{c r}^{\prime}(G)+\gamma(G)+1 \leq p+$ $\gamma_{c}^{\prime}(G)$.

Theorem 2.13: For any graph $(p, q)$ with $p \geq 3$ vertices,

$$
\gamma_{c r}^{\prime}(G) \geq\left\lceil\frac{\operatorname{diam}(G)+1}{2}\right\rceil-1 .
$$


Proof: Let $E=\left\{e_{1}, e_{2}, \ldots \ldots, e_{n}\right\} \subseteq E(G)$ be the set of edges which constitute the longest path between two distinct vertices $u, v \in V(G)$ such that $d(u, v)=\operatorname{diam}(G)$. Now $E_{1} \subseteq$ $E(G), \forall e_{i} \in E_{1}$ since $E_{1}$ be the minimal set of edges which covers all the edges in $G$ then $E_{1}$ is a minimal edge dominating set of $G$. Further if $\operatorname{deg}\left(e_{j}\right) \geq 1, e_{j} \in E(G)-E_{1}$ then $\left\langle E(G)-E_{1}\right\rangle$ is regular then $\left\{E_{1}\right\}$ is a coregular edge dominating set. It follows that $\left|E_{1}\right| \geq\left|\frac{\operatorname{diam}(G)+1}{2}\right|-1$. Hence $\gamma_{c r}^{\prime}(G) \geq\left\lceil\frac{\operatorname{diam}(G)+1}{2}\right\rceil-1$.

\section{REFERENCES}

[1] Araya chaemchan, The edge domination number of connected graphs, Australasian Journal of Combinatorics, Vol 48, 185-189.

[2] S. Arumugam and S. Velammal, 1998. Edge domination in graphs, Taiwanese J. of Mathematics,2(2), $173-179$.

[3] S. Arumugam and S. Velammal, 2009. Connected edge domination in graphs, Allahabad Mathematical Society, Vol 24,part, 43-49.

[4] C. Berge, 1962. Theory of graphs and its applications, Methuen, London,.

[5] E. J. Cockayne, R. M. Dawes and S. T. Hedetniemi, 1980. Total domination in graphs, Networks, 10, 211 219.

[6] G. S. Domke, J. H. Hattingh, S. T. Hedetniemi, R. C. Laskar and L. R. Markus, 1997. Restrained domination in graphs, Discrete Math., 203, $61-69$.

[7] F. Harary, 1969. Graph theory, Adison Wesley, Reading Mass.

[8] T.W. Haynes, S. T. Hedetniemi and P. J. Slater, 1997. Domination in graphs: Advanced topics, Marcel Dekker, Inc., New York.

[9] F. Harary and T. W. Haynes, 2000. Double domination in graphs Ars Combin Vol 55, 201-213.

[10] V. R. Kulli, 2010. Theory of domination in graph, Vishwa international publications, India.

[11] S. Mitchell and S. T. Hedetniemi, 1977. Edge domination in trees, Congr. Numer, 19, $489-509$.

[12] M. H. Muddebihal and Priyanka .H. Mandarvadkar, 2019. Co-regular split domination in graphs. JES, Vol$10,259-264$.

[13] M. H. Muddebihal and Priyanka. H. Mandarvadkar, 2020. Co-regular restrained domination in graphs. CEJ,Vol -11, 236-241.

[14] M. H. Muddebihal and Priyanka. H. Mandarvadkar, 2020. Co-regular total domination in graphs. International Journal of Applied Information Systems (IJAIS) Vol-12 16-19.

[15] O.Ore,1962. Theory of graphs, Amer. Math.Soc.Colloq.Publ. 38. Providence, RI. 